\title{
Grip strength and exposure to hue differences in visual stimuli: Is postural status a factor?
}

\author{
ROBERT J. PELLEGRINI, ALEXANDER G. SCHAUSS, T. J. KERR, and BART K. AH YOU \\ San Jose State University, San Jose, California 95192
}

\begin{abstract}
In previous experiments investigating effects of color on muscle strength, subjects were seated during the test procedure, and although statistically significant, magnitude of color effect differences were very small. So as to explore the possible role of organismic state variables associated with body position, subjects in the present study were tested while standing. This time, no significant difference was found between hand dynamometer squeeze scores of 36 high school wrestlers as they stared at a pink, compared with a blue, stimulus card. Directions for future inquiry into muscle-specific loci of kinesiological color effects are considered. But the lack of unambiguously established experimental evidence as to the reliable effects of color on muscle strength is underscored.
\end{abstract}

Can skeletal muscle strength, as Ott (1979) has suggested, be affected by the hue of visual stimuli to which a person is exposed? Until recently, the only affirmative empirical evidence to this effect was derived from the so-called "kinesoid test," in which a subject holds his arm straight out in front of him and tries to resist as vigorously as possible a demonstrator's efforts to push the arm down. Following establishment of a "baseline," loss of strength is typically observed when the test is repeated immediately thereafter while a pink card is held about 15 in. in front of the subject's eyes, with a recovery in resistance shown when a blue card is then similarly placed (Schauss, 1979). Such demonstrations, however, provide a most unsatisfactory basis of inference, in that the strength measure used depends entirely upon impressions of the subject's resistance, as judged by a not necessarily impartial demonstrator with a considerable leverage advantage.

In the only conventional experimental tests of the "kinesoid hypothesis" yet reported, significantly greater strength responses were indeed found to occur as subjects stared at a blue, compared with a pink, stimulus card, using hand dynamometer squeeze scores (Pellegrini \& Schauss, 1980) and quadriceps femoris extension (Pellegrini, Schauss, \& Birk, 1980) as the dependent measures. Although substantially greater in the latter than in the former situation, magnitude of the observed effect in both experiments was so limited as to leave the issue still very much in doubt.

The question arises as to why the kinesoid test should yield apparently more unambiguous outcomes

This study was supported in part by grants to the first author from the American Institute for Biosocial Research and the San Jose State University Foundation. Requests for reprints should be sent to Robert J. Pellegrini, Department of Psychology, San Jose State University, San Jose, California 95192 A. G. Schauss is the director of the American Institute for Biosocial Research, Tacoma, Washington 98466. than do controlled laboratory studies such as those described above. One possibility is that results of the kinesoid demonstration are attributable largely to confounding sources of variance, such as demonstrator bias, demand characteristics affecting subjects' expectations, and so on (see Pellegrini \& Schauss, 1980; Pellegrini et al., 1980). Another possibility is that the laboratory procedures have not adequately represented the conditions required to produce the effect. For one thing, as noted by a number of critics of the previous experimental studies, the laboratory subjects performed the strength-assessment task while seated, whereas the kinesoid test is conducted with the subject standing.

It is conceivable, albeit by no means obvious why it should be so, that postural status or body tonus characteristics might be associated with differences in organismic state that somehow operate to minimize or enhance the color-strength effect. The present study was thus designed to investigate magnitude of color effects on the grip strength of subjects tested while standing, rather than sitting, as was the case in the first experiment (Pellegrini \& Schauss, 1980).

\section{METHOD}

\section{Subjects}

The subjects were 36 right-handed male high school students between 14 and 17 years old, selected at random from a group of participants in a summer wrestling camp.

\section{Materials \\ The materials used were: a Lafayette hand dynamometer, a Brenet stopwatch, two $18 \times 24$ in. cardboard plates (one pink and one blue), and a specially constructed aluminum tubular view-frame apparatus ${ }^{1}$ made to rest on the subject's chest and shoulders so as to hold the stimulus plates 12 to 15 in. in front of his eyes.}

\section{Procedure}

All subjects were tested individually, standing upright throughout the experiment in a quiet, windowless area of the 
gymnasium lighted by a 150-W incandescent bulb. Upon the subject's arrival at the test location, the dynamometer was placed in the subject's right (preferred) hand, and he was instructed to let it hang relaxed by his side in a grip position until he was told to squeeze it. The view frame was then set in place over the subject's head, one of the cardboard plates was inserted into position, and the subject was instructed to stare at the middle of the card until told to stop. Exactly $60 \mathrm{sec}$ later the subject was told to continue staring at the card, to squeeze the handle in his right hand, and to give the handle back to the experimenter after squeezing it as hard as possible. After being handed the dynamometer by the subject, the experimenter then removed the first card from the view frame, recorded to the nearest $.5 \mathrm{~kg}$ the grip strength score indicated, and returned the dynamometer to the subject, instructing him to hold it relaxed by his side, as before. Following a $60-\mathrm{sec}$ rest period, this procedure was repeated for the other color plate. Both plates were concealed from the subject until they were presented in the view frame. Order effects were balanced by randomly assigning 18 subjects to a blue-pink and 18 subjects to a pink-blue presentation order condition.

\section{RESULTS AND DISCUSSION}

A two-tailed $t$ test for matched samples revealed no significant difference [ $\mathrm{t}(35)=1.07$ ] between the hand dynamometer scores (in kilograms) obtained during exposure of the pink (mean $=31.22, \mathrm{SD}=7.22$ ), compared with the blue (mean $=30.68, \mathrm{SD}=6.36$ ), card. Taken together with the previous study measuring grip response (Pellegrini \& Schauss, 1980), these results thus fail to support the view that postural status (i.e., sitting vs. standing) may be a factor mediating kinesio- logical effects of color exposure. These findings also raise serious doubts about the general validity of the kinesoid hypothesis. One question that does merit further inquiry is whether the functions of some muscle groups (e.g., the deltoid) might be more accessible to such manipulation than are others. But for the time being, experimental data indicating unambiguously reliable effects of color on muscle strength remain to be established.

\section{REFERENCES}

OTт, J. N. The dual function of the eyes. Southern Journal of Optometry, 1979, 21, 8-13.

Pellegrini, R. J., \& Schauss, A. G. Muscle strength as a function of exposure to hue differences in visual stimuli: An experimental test of the kinesoid hypothesis. Journal of Orthomolecular Psychiatry, 1980, 9, 144-147.

Pellegrini, R. J., Schauss, A. G., \& Birk, T. J. Leg strength as a function of exposure to visual stimuli of different hues. Bulletin of the Psychonomic Society, 1980, 16, 111-112.

Schauss, A. G. Tranquilizing effect of color reduces aggressive behavior and potential violence. Journal of Orthomolecular Psychiatry, 1979, 8, 218-220.

\section{NOTE}

1. Thanks are due to John Empey, San Jose State University Psychology Laboratory Technician, for the design and construction of this device.

(Received for publication December 15, 1980.) 